\title{
Controlling the Amorphous and Crystalline State of Multinary Alloy Nanoparticles in An Ionic Liquid
}

\author{
Alba Garzón-Manjón ${ }^{1,+}{ }^{\dagger}$ Hajo Meyer ${ }^{2,+}{ }^{+}$Dario Grochla ${ }^{2}$, Tobias Löffler ${ }^{3}{ }^{(1)}$, \\ Wolfgang Schuhmann ${ }^{3}(\mathbb{D})$, Alfred Ludwig ${ }^{2}$ and Christina Scheu ${ }^{1, *(\mathbb{D}}$ \\ 1 Max-Planck-Institut für Eisenforschung GmbH, Max-Planck-Straße 1, 40237 Düsseldorf, Germany; \\ a.garzon@mpie.de \\ 2 Werkstoffe der Mikrotechnik, Institut für Werkstoffe, Fakultät für Maschinenbau, Ruhr-Universität Bochum, \\ Universitätsstr.150, D-44801 Bochum, Germany; hajo.meyer@ruhr-uni-bochum.de (H.M.); \\ dario.grochla@rub.de (D.G.); alfred.ludwig@ruhr-uni-bochum.de (A.L.) \\ 3 Analytical Chemistry - Center for Electrochemical Sciences (CES), Faculty of Chemistry and Biochemistry, \\ Ruhr University Bochum, Universitätsstr. 150, D-44780 Bochum, Germany; \\ jan.loeffler@ruhr-uni-bochum.de (T.L.); wolfgang.schuhmann@rub.de (W.S.) \\ * Correspondence: scheu@mpie.de; Tel.: +49-(0)211-6792720 \\ + These authors contributed equally to this work
}

Received: 15 October 2018; Accepted: 1 November 2018; Published: 4 November 2018

check for updates

\begin{abstract}
Controlling the amorphous or crystalline state of multinary Cr-Mn-Fe-Co-Ni alloy nanoparticles with sizes in the range between $\sim 1.7$ and $\sim 4.8 \mathrm{~nm}$ is achieved using three processing routes. Direct current sputtering from an alloy target in the ionic liquid 1-butyl-3-methylimidazolium bis(trifluoromethylsulfonyl)imide leads to amorphous nanoparticles as observed by high-resolution transmission electron microscopy. Crystalline nanoparticles can be achieved in situ in a transmission electron microscope by exposure to an electron beam, ex situ by heating in vacuum, or directly during synthesis by using a high-power impulse magnetron sputtering process. Growth of the nanoparticles with respect to the amorphous particles was observed. Furthermore, the crystal structure can be manipulated by the processing conditions. For example, a body-centered cubic structure is formed during in situ electron beam crystallization while longer ex situ annealing induces a face-centered cubic structure.
\end{abstract}

Keywords: multinary alloy nanoparticle; ionic liquid; dc sputtering; HiPIMS sputtering; nanoparticle growth; in situ TEM

\section{Introduction}

Multinary metal nanoparticles (NPs) have attracted increasing interest over the past years for a multitude of applications in optics [1,2], electronics [3], catalysis [4] or other industrial fields [5] due to their unique and tailorable properties [6-8]. Synthesis methods for NPs can be divided into bottom-up and top-down processes [9]. The bottom-up processes, using the reduction or decomposition of chemical precursors, allow to achieve a wide variety of NPs. However, the synthesis of multinary NPs is increasingly complicated with the increasing number of constituents and decomposition of by-products, which remain in the suspension and might cause stability issues [10]. As pointed out by Chen et al. [11], there is a large demand for explorative synthesis approaches and the in-depth analysis of multinary NPs as these might possess exceptional properties.

Physical vapor deposition (PVD), as a top-down technique, allow to prepare NPs by sputtering the elements into ionic liquids (IL) [12]. The ILs act as media for growth and due to their low vapor pressures and melting points, they allow to control the size and shape of the NPs [13-15]. Synthesis of 
metal NPs via sputtering in ionic liquids to produce single element NPs $[16,17]$ or alloy NPs $[18,19]$ and techniques for transfer and accumulation [20,21] have been reported [22]. Co-sputtering from elemental or alloy targets in ILs has almost no limits in the combination of different constituents in multinary NPs and provides a high control in the elemental composition [11,22-24]. Still, the formation process in or on the IL itself is not fully understood [13,25].

High-power impulse magnetron sputtering (HiPIMS) shows several advantages over conventional direct current $(\mathrm{dc})$ magnetron sputtering, where the sputtered vapor consists mainly of neutral species. HiPIMS provides a high plasma density and a high ionization fraction of the sputtered species by up to $90 \%$ [26]. It allows for more control of the thin film growth by controlling the energy and direction of the sputtered species and opens new pathways for the synthesis of multinary NPs.

In this research work, the formation of quinary alloy Cr-Mn-Fe-Co-Ni NPs by sputtering into IL has been studied to understand in the nanoscale range their excellent catalytic behavior in oxygen reduction reaction (ORR). An intrinsic activity comparable to $\mathrm{Pt}$ was recently reported by Löffler et al. [27]. Furthermore, 1-butyl-3-methylimidazolium bis(trifluoromethylsulfonyl)imide IL was used as a stabilizer and suspension media at the same time due to its high stability against decomposition generating stable nanoparticles, as was observed by Meyer et al. [16]. Moreover, it can be utilized in a high vacuum state which has to be applied during sputtering. After conventional dc magnetron sputtering, no crystalline NPs were observed by transmission electron microscopy (TEM) and thus, we classified these as-deposited NPs as amorphous. To obtain crystalline NPs, three processing routes were used: (i) in situ electron beam irradiation, (ii) ex situ heating, and (iii) HiPIMS.

Aberration-corrected high-resolution TEM (HRTEM) and high-resolution scanning transmission electron microscopy (HRSTEM) coupled with energy-dispersive X-ray spectroscopy (EDS) were used to study the effect of the processing routes on the crystallinity, as well as on the size, shape, and morphology of the multinary NPs.

\section{Materials and Methods}

\subsection{Materials}

For the synthesis of NPs by sputtering into IL, 1-butyl-3-methylimidazolium bis(trifluoromethylsulfonyl)imide (purity $>99 \%$, [Bmim][(Tf) $\left.{ }_{2} \mathrm{~N}\right]$ ) was purchased from IoLiTec-Ionic Liquids Technologies GmbH (Heilbronn, Germany) (specified impurities were halides (IC) < 100 ppm, and water $(\mathrm{KF})<71 \mathrm{ppm})$. The IL was stored under an Ar atmosphere and was used with no further purification.

The custom made alloy sputter target $\mathrm{Cr}-\mathrm{Mn}-\mathrm{Fe}-\mathrm{Co}-\mathrm{Ni}$ had a purity of $99.95 \%$ and a composition of $\mathrm{Cr}_{18} \mathrm{Mn}_{20} \mathrm{Fe}_{20} \mathrm{Co}_{21} \mathrm{Ni}_{21}$ as analyzed by $\mathrm{X}$-ray fluorescence.

\subsection{Sputter Deposition}

The synthesis was performed in a magnetron sputter system (AJA POLARIS-5 chamber from AJA International Inc., Country Way North Scituate, MA, USA) with 1.5" diameter cathodes and a dc power supply (DC-XS 1500). For HIPIMS deposition, a SIPP 2000 USB 10-300-D with an ADL Generator (GS15) from Melec GmbH (Baden-Baden, Germany) was applied. Ar was used as the process gas with a purity of $99.999 \%$.

A multiple cavity holder was applied for sputtering into the IL with a volume of $40 \mu \mathrm{L}$ per cavity as described elsewhere [16]. With specially designed covers, either 6 or a maximum of 64 cavities are available. For cleaning, the holder was ultrasonicated $20 \mathrm{~min}$ each in acetone and isopropanol. The cavities were filled with the IL under an Ar atmosphere and transferred directly into the deposition chamber. After deposition, the holder was removed and transported in an Ar atmosphere and the IL was collected and stored under Ar.

The DC sputter deposition of Cr-Mn-Fe-Co-Ni NPs was performed as follows: the IL $[\mathrm{Bmim}]\left[(\mathrm{Tf})_{2} \mathrm{~N}\right]$ and pieces of a patterned $\mathrm{Si} / \mathrm{SiO}_{2}$ wafer $(2 \mathrm{~cm} \times 3 \mathrm{~cm}$, photolithographically structured 
with a photoresist lift-off cross pattern for film thickness determination) were stored in the vacuum chamber overnight resulting in a start vacuum of $1.7 \times 10^{-4} \mathrm{~Pa}$. After plasma ignition at $1.33 \mathrm{~Pa}$, $20 \mathrm{~W}$ and a $2 \mathrm{~min}$ precleaning step, the Ar pressure was reduced to $0.5 \mathrm{~Pa}$. A cathode tilt of $12^{\circ}$ of the cathode's normal respective to the normal of the cavity holder was used for the deposition. The deposition was performed at $30 \mathrm{~W}(312 \mathrm{~V}, 95 \mathrm{~mA})$ for $2 \mathrm{~h}$.

HiPIMS sputter deposition of Cr-Mn-Fe-Co-Ni NPs was carried out as follows: The initial steps were the same as described above for dc sputtering with a starting vacuum of $3.0 \times 10^{-4} \mathrm{~Pa}$ after overnight pumping. To ensure an arc-free and stable HiPIMS plasma, the target ignition was performed gradually. Starting with $400 \mathrm{~Hz}, 40 \mathrm{~W}, 30 \mathrm{~s}$ (on 40/ off 2560) followed by $200 \mathrm{~Hz}, 40 \mathrm{~W}$, $30 \mathrm{~s}$ (on 40/ off 4960) and finally $100 \mathrm{~Hz}, 40 \mathrm{~W}, 30 \mathrm{~s}$ (on 40 / off 9960) at an Ar pressure of $2.66 \mathrm{~Pa}$. The HiPIMS deposition was performed with $100 \mathrm{~Hz}$, on $40 /$ off 9960, 0.5 Pa, average power $\mathrm{P}_{\mathrm{m}}=40 \mathrm{~W}$ $\left(670 \mathrm{~V}, 30 \mathrm{~mA}\right.$, peak current $\mathrm{A}_{\mathrm{p}}=27 \mathrm{~A}$, peak current density $2.37 \mathrm{Acm}^{-2}$ and a peak power density $1.59 \mathrm{kWcm}^{-2}$ ) for $3 \mathrm{~h}$ providing a film thickness of $270 \mathrm{~nm}$ on the patterned reference regions of the wafer.

The multinary dc-as-deposited NPs in the IL were heat treated ex situ under a vacuum (30 Pa) at $100{ }^{\circ} \mathrm{C}$ for $2,5,10$, and $15 \mathrm{~h}$ in an oil bath and then cooled down to room temperature in order to verify a possible crystallization.

\subsection{Materials Characterization}

For TEM investigations, a holey carbon-coated gold grid (Plano GmbH, Wetzlar, Germany) was used, on which a $2.5 \mu \mathrm{L} \mathrm{NP} / \mathrm{IL}$ sample was dropped on the coated side and left for adhesion for $2 \mathrm{~h}$. Dried acetonitrile was used to wash the grid dropwise for $1 \mathrm{~h}$ under inert conditions. The grid was stored in an Ar atmosphere. Further information can be found elsewhere [16].

TEM characterization of multinary NPs was performed in two Titan Themis 60-300 X-FEG (Thermo Fischer Scientific, Eindhoven, Netherlands) equipped either with an image corrector or a probe corrector, both operated at $300 \mathrm{kV}$. TEM images were recorded on a metal-oxide-semiconductor (CMOS) camera with $4 \mathrm{k} \times 4 \mathrm{k}$ pixels. High angle annular dark field (HAADF) images (72-352 mrad) and EDS measurements were carried out using STEM with currents of $\sim 90 \mathrm{pA}$ and $\sim 150$ pA respectively, a convergence semi-angle of $\sim 23.8 \mathrm{mrad}$ and a beam size of $\sim 0.1 \mathrm{~nm}$. For each sample, up to $200 \mathrm{NPs}$ were investigated.

Beam-induced in situ crystallization of the multinary NPs was achieved at $300 \mathrm{kV}$ with a dose rate $\sim 1.2 \times 10^{4} \mathrm{e} / \mathrm{nm}^{2} \mathrm{~s}$, for a total time of $40 \mathrm{~min}$. Crystallization was also possible by using a $200 \mathrm{kV}$ electron beam. To investigate the crystallinity, Fast Fourier transforms (FFT) were calculated from the HRTEM images, always averaging over several NPs. Several NPs contribute to the FFT which was calculated using images of $33.5 \times 33.5 \mathrm{~nm}^{2}$.

ICP MS analysis was performed with an iCAP RQ ICP MS (Thermo Scientific, Waltham, Massachusetts, USA) employing a TELEDYNE CETAC technologies ASX 560 autosampler. Calibration of elemental concentration was performed with a Q/Qnova calibration solution (Thermo Scientific, Waltham, Massachusetts, USA) in the std mode. A total of $300 \mu \mathrm{L}$ suspension of sputtered NPs in IL have been dissolved in $69 \% \mathrm{HNO}_{3}$ (ROTIPURAN Supra of Roth) and diluted with $\mathrm{H}_{2} \mathrm{O}$ to an elemental concentration of approximately 100-200 ppb before transfer to the plasma. Water was purified using an SG purification system yielding a conductivity of $0.055 \mu \mathrm{S} \mathrm{cm}-1$.

\section{Results}

DC sputtering from the Cr-Mn-Fe-Co-Ni target into the IL results in amorphous NPs (see Figure 1a) as no lattice fringes and no reflections in the FFT were observed. Thus, the NPs in the initial state after dc sputtering are referred to as amorphous in the following. For the formation of crystalline multinary NPs, additional processing routes were investigated. 


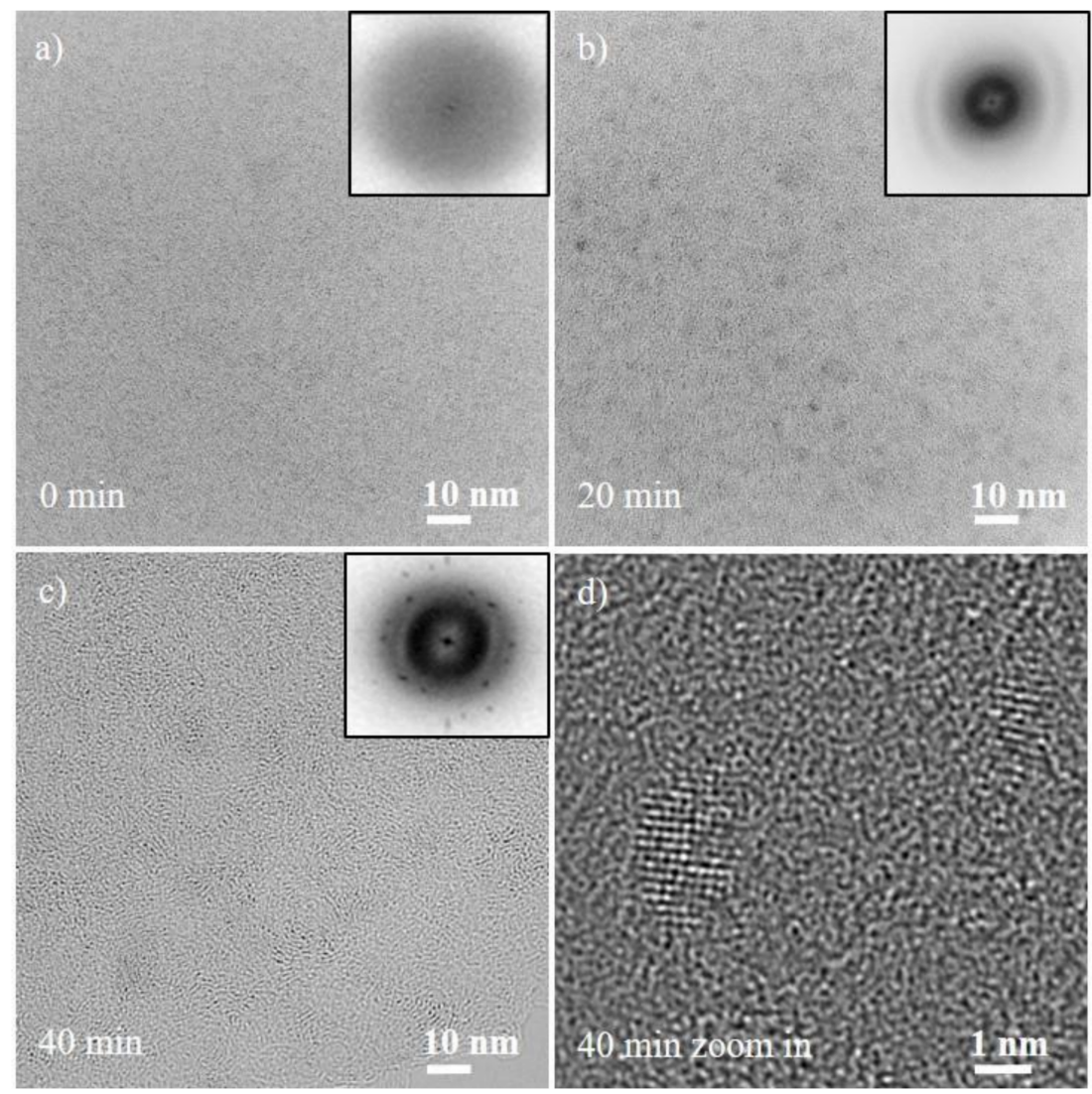

Figure 1. Results of the in situ TEM crystallization experiment for dc-sputtered multinary Cr-Mn-Fe-Co-Ni NPs transferred on a carbon coated gold grid from the IL. (a) shows amorphous as-deposited NPs. (b) and (c) display TEM images and FFT patterns (shown as insets) after 20 and 40 min electron beam illumination and the resulting crystallized NPs. (d) HRTEM image of multinary NPs showing lattice fringes. For better visibility, the contrast in the FFT patterns is inverted.

Electron-beam-induced crystallization was carried out to transform the NPs from the amorphous to the crystalline state as shown in Figure 1. After $20 \mathrm{~min}$ of electron beam irradiation, the NPs are still amorphous (Figure 1b). Electron beam irradiation of the NPs for more than 40 min leads to a structural transformation and formation of a crystalline state. As a long-range order has been generated, lattice fringes can be observed and reflection spots are present in the FFT (Figure 1c). The atomic arrangement is visible in Figure 1d which shows two NPs at high magnification after the in situ crystallization process.

The phase transformation of the NPs from the amorphous to crystalline state was studied by analyzing the FFT patterns at different stages. At the initial stage $(0 \mathrm{~min})$, no reflections are observed. After $20 \mathrm{~min}$, the TEM image was recorded out of focus to get more contrast between the amorphous NPs and the amorphous carbon film to enable the study of the size and the morphology of the NPs in this state. The rings observed in the FFT corresponds to the carbon support. After 40 min of electron beam illumination of the NPs, diffraction spots from the transformation of the NPs to the crystalline state were observed in the FFT (Figure 1c). The reflections can be assigned to the body-centered cubic (bcc) structure: the first ring corresponds to $d_{1}=0.205 \mathrm{~nm}$ and is related to the $\{011\}$ planes and the second ring $\mathrm{d}_{2}=0.145 \mathrm{~nm}$ to the $\{002\}$ planes.

The size distribution of the NPs at different stages of the electron-beam assisted crystallization process shows an increase in the diameter in size from $1.7 \pm 0.2 \mathrm{~nm}$ (amorphous) to $2.6 \pm 0.3 \mathrm{~nm}$ (crystalline). This increased diameter can be related to the crystallization process and to Ostwald ripening [28]. The histogram of the NPs size distribution is given in the supplementary information (SI) in Figure S1. The NPs are facetted and have a nearly equiaxed shape. 
As an alternative approach to electron-beam-induced crystallization of the dc-as-deposited amorphous NPs, ex situ heating was carried out under vacuum $(30 \mathrm{~Pa})$ at $100{ }^{\circ} \mathrm{C}$ for $2,5,10$ and $15 \mathrm{~h}$ to induce crystallization. The crystal structure was studied using FFT instead of selected area diffraction to avoid the high contribution from the amorphous signal of the carbon-coated TEM grid.

After two hours of annealing at $100{ }^{\circ} \mathrm{C}$, NPs increase slightly in size (1.9 $\left.\pm 0.2 \mathrm{~nm}\right)$ and an ordering process starts for the NPs which become crystalline as is visible in their lattice fringes in Figure 2a. After $5 \mathrm{~h}$ of annealing, a further increase in order is generated confirmed by the FFT pattern in Figure $2 b$ where more diffraction spots are obtained. The NPs are still $1.9 \pm 0.2 \mathrm{~nm}$ in diameter. After $10 \mathrm{~h}$, all NPs are crystalline (Figure 2c) and have grown further to $2.4 \pm 0.4 \mathrm{~nm}$ in diameter. For annealing times up to $10 \mathrm{~h}$, only the bcc crystal structure is observed. The first ring from the FFT patterns corresponds to $\{011\}\left(\mathrm{d}_{1}=0.205 \mathrm{~nm}\right)$ and the second to $\{002\}\left(\mathrm{d}_{2}=0.145 \mathrm{~nm}\right)$. The shape of the NP is similar to the in situ case.
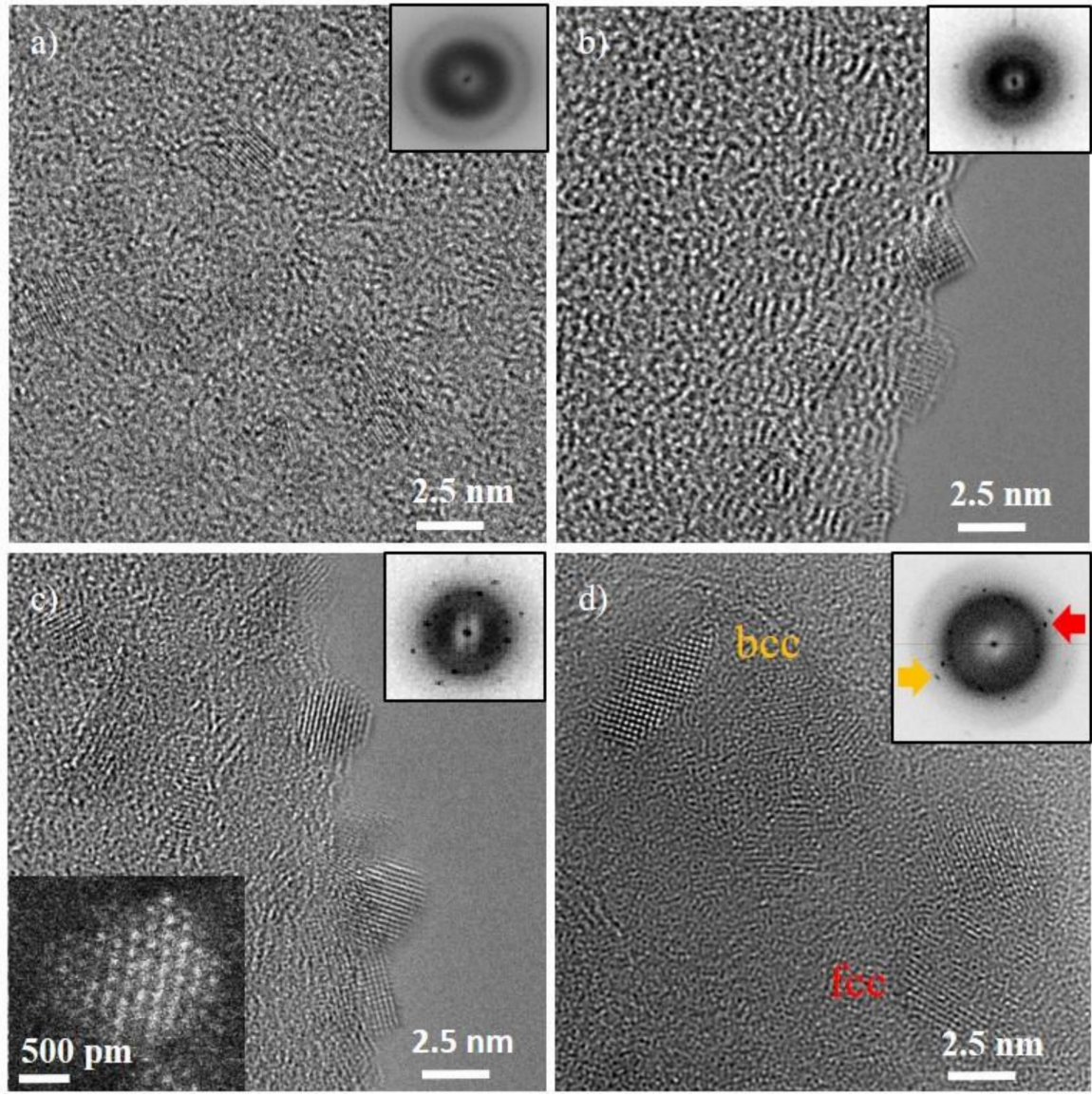

Figure 2. Results of the TEM analysis of Cr-Mn-Fe-Co-Ni NPs after ex situ crystallization at $100^{\circ} \mathrm{C}$ under vacuum (30 Pa) for (a) $2 \mathrm{~h}$, (b) $5 \mathrm{~h}$, (c) $10 \mathrm{~h}$, and (d) $15 \mathrm{~h}$. FFT patterns (insets) show the evolution of the NPs crystallinity. In (a-c) all NPs possess the bcc structure and in (d) additional reflections from the fcc phase were found. A HAADF STEM image of one NP is shown in the inset of (c). The arrows in (d) point to specific reflections of fcc and bcc which can be clearly distinguished.

After heating the multinary IL NP suspension for $15 \mathrm{~h}$ at $100^{\circ} \mathrm{C}$ under vacuum, the formation of a phase mixture between bcc and the face-centered cubic (fcc) is observed. The first reflections which are expected for fcc $\{111\}\left(d_{1}=0.208 \mathrm{~nm}\right)$ are overlapping with the first ring from bcc $\{011\}\left(\mathrm{d}_{1}=0.205 \mathrm{~nm}\right)$. The presences of an fcc phase is proven with the second set of reflections which belong to a lattice plane distance $d_{2}=0.180 \mathrm{~nm}$. This value corresponds to the $\{002\}$ planes of an fcc crystal structure. The NPs have grown further to a diameter of $4.8 \pm 0.8 \mathrm{~nm}$ as shown in the histogram of the NPs in Figure S2. To understand this change in crystal structure, EDS was performed as discussed below. 
With HiPIMS, crystalline NPs are obtained already in the as-deposited state, which also show the biggest diameter of $3.2 \pm 0.5 \mathrm{~nm}$. The histogram is provided in the SI (Figure S3). Consistently, they are also facetted and nearly equiaxed. The analysis of the FFT patterns reveals that these NP possess the bcc crystal structure (Figure 3).


Figure 3. (a) TEM image of HiPIMS sputtered Cr-Mn-Fe-Co-Ni NPs transferred on a carbon-coated gold grid from the IL and its corresponding FFT (shown as an inset). (b) HRTEM shows bcc lattice planes within the NPs.

EDS analysis in the STEM mode was used to identify and quantify the composition of NPs and to understand the reason for the change from a bcc to fcc structure. The color-coded elemental maps in Figure 4 represent the different chemical elements present in NPs after in situ electron beam crystallization. STEM-EDS requires that the NPs are stable on the grid for a certain time to gain enough counts for a precise single NP quantification. However, NPs were not stable under these conditions. Therefore, maps were recorded with a spatial resolution of $\sim 0.5 \mathrm{~nm}$ as exemplarily shown in Figure 4 . No individual atomic columns are resolved. In addition, carbon contamination from the support grid and the surrounding IL prevents higher spatial resolution in the EDS maps. Nevertheless, we were able to analyze the changes in the chemical composition of the NPs prepared by the different processes.
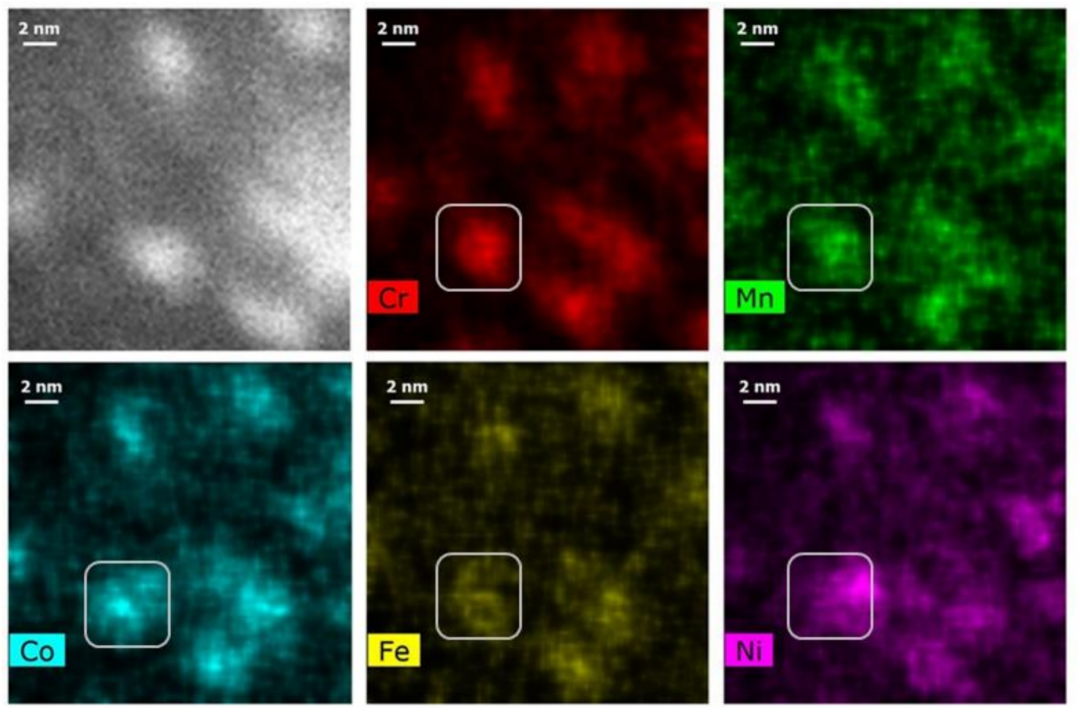

Figure 4. Chemical composition analysis of Cr-Mn-Fe-Co-Ni NPs transferred from the NPs IL suspension onto a carbon-coated gold grid and crystallized with the electron beam. The STEM-EDS maps show the presence and homogeneous distribution of $\mathrm{Cr}, \mathrm{Mn}, \mathrm{Fe}, \mathrm{Co}$, and Ni in each NP. For determination of the atomic composition of single NPs, specific areas were chosen as shown exemplary with a grey rectangle. 
The EDS data were analyzed using the Cliff-Lorimer equation [29] and the analysis was done for both, individual NPs and for areas including NPs and their surroundings. The K factors used for the quantification were determined using a reference from bulk $\mathrm{Cr}-\mathrm{Mn}$-Fe-Co-Ni material in order to achieve higher accuracy.

The elemental composition of single multinary NPs with a bcc crystal structure achieved by dc sputtering and electron beam assisted crystallization have according to the STEM-EDS analysis the following range: $\mathrm{Cr} 33-46$ at $\%$, Mn 1-3 at $\%$, Fe 11-15 at $\%$, Co 23-26 at $\%$, and Ni 18-30 at $\%$ (see Table 1). After ex situ heating for $15 \mathrm{~h}$ at $100{ }^{\circ} \mathrm{C}$, NPs are generated with either a bcc or fcc crystalline structure. Their elemental composition ranges between $\mathrm{Cr} 17-27$ at \%, Mn 5-8 at\%, Fe 15-17 at\%, Co 31-36 at \%, and Ni 19-24 at\% (see Table 1). The content of Mn, Fe, and Co is similar within the individual NP while the amount of $\mathrm{Cr}$ and $\mathrm{Ni}$ is differing more strongly. This might be related to the local inhomogeneity of the IL with different tendency to attract or reject these elements. Further studies will clarify this point.

Table 1. Composition range of dc sputtered (as-deposited and $15 \mathrm{~h}$ annealed) and HiPIMS sputtered NPs transferred from the NP IL suspension onto a carbon-coated gold grid. Comparison of the STEM-EDS analysis with the overall composition of the sputtered NPs within the IL measured with ICP-MS $\left({ }^{*}\right)$ in at $\%$.

\begin{tabular}{cccccc}
\hline \multirow{2}{*}{ Element } & \multicolumn{3}{c}{ Dc Sputtered } & \multicolumn{2}{c}{ HiPIMS Sputtered } \\
\cline { 2 - 6 } & $\begin{array}{c}\text { As-Deposited } \\
\text { in at } \%\end{array}$ & $\begin{array}{c}\text { 15 h of Annealed } \\
\text { in at } \%\end{array}$ & Overall in at $\%$ & $\begin{array}{c}\text { As-Deposited } \\
\text { in at } \%\end{array}$ & Overall in at\% \\
\hline $\mathrm{Cr}$ & $33-46$ & $17-27$ & $31^{*}$ & $15-20$ & $22^{*}$ \\
$\mathrm{Mn}$ & $1-3$ & $5-8$ & $4^{*}$ & $10-19$ & $15^{*}$ \\
$\mathrm{Fe}$ & $11-15$ & $15-17$ & $11^{*}$ & $19-26$ & $16^{*}$ \\
$\mathrm{Co}$ & $23-26$ & $31-36$ & $23^{*}$ & $26-31$ & $22^{*}$ \\
$\mathrm{Ni}$ & $18-30$ & $19-24$ & $31^{*}$ & $13-21$ & $25^{*}$ \\
\hline
\end{tabular}

ICP-MS measurements reveal an overall composition in the DC sputtered IL of Cr 31 at\%, Mn 4 at $\%$, Fe 11 at \%, Co 23 at\%, and Ni 31 at\% (see Table 1). While the Mn, Fe, and Co contents agree well with the STEM-EDS results, the $\mathrm{Cr}$ and Ni content are higher in the ICP-MS measurements. This can be explained as follows: during the ICP-MS analysis, all the elements present in the solution are detected. This means that single clusters from the different elements which are not incorporated in the nanoparticles but located within the IL are also taken into account.

The NPs synthesized using HiPIMS show an elemental composition between Cr 15-20 at\%, Mn 10-19 at\%, Fe 19-26 at\%, Co 26-31 at\% and Ni 13-21 at\%. These values obtained by STEM-EDS are also consistent with the ICP-MS of the sample consisting of the NPs and the surrounding IL which still contains some of the sputtered species. The overall composition determined by ICP-MS is Cr 22 at $\%$, Mn 15 at $\%$, Fe 16 at\%, Co 22 at $\%$ and Ni 25 at\%, as shown in Table 1.

\section{Discussion}

In the present study we demonstrated that with sputtering into ILs, multinary NPs with sizes ranging from 2 to $4 \mathrm{~nm}$ can be achieved, in both the amorphous or crystalline state. Our multinary NPs prepared using the IL synthesis strategy are much smaller compared to the ones of Chen et al [11]. The small size makes them particularly suitable in different fields such as dye degradation [30], hydrogen evolution [31], methanol oxidation [32] or as ORR catalyst [27].

The effects of the different sputtering routes (HiPIMS versus DC) and post-deposition treatments are summarized in Figure 5. 


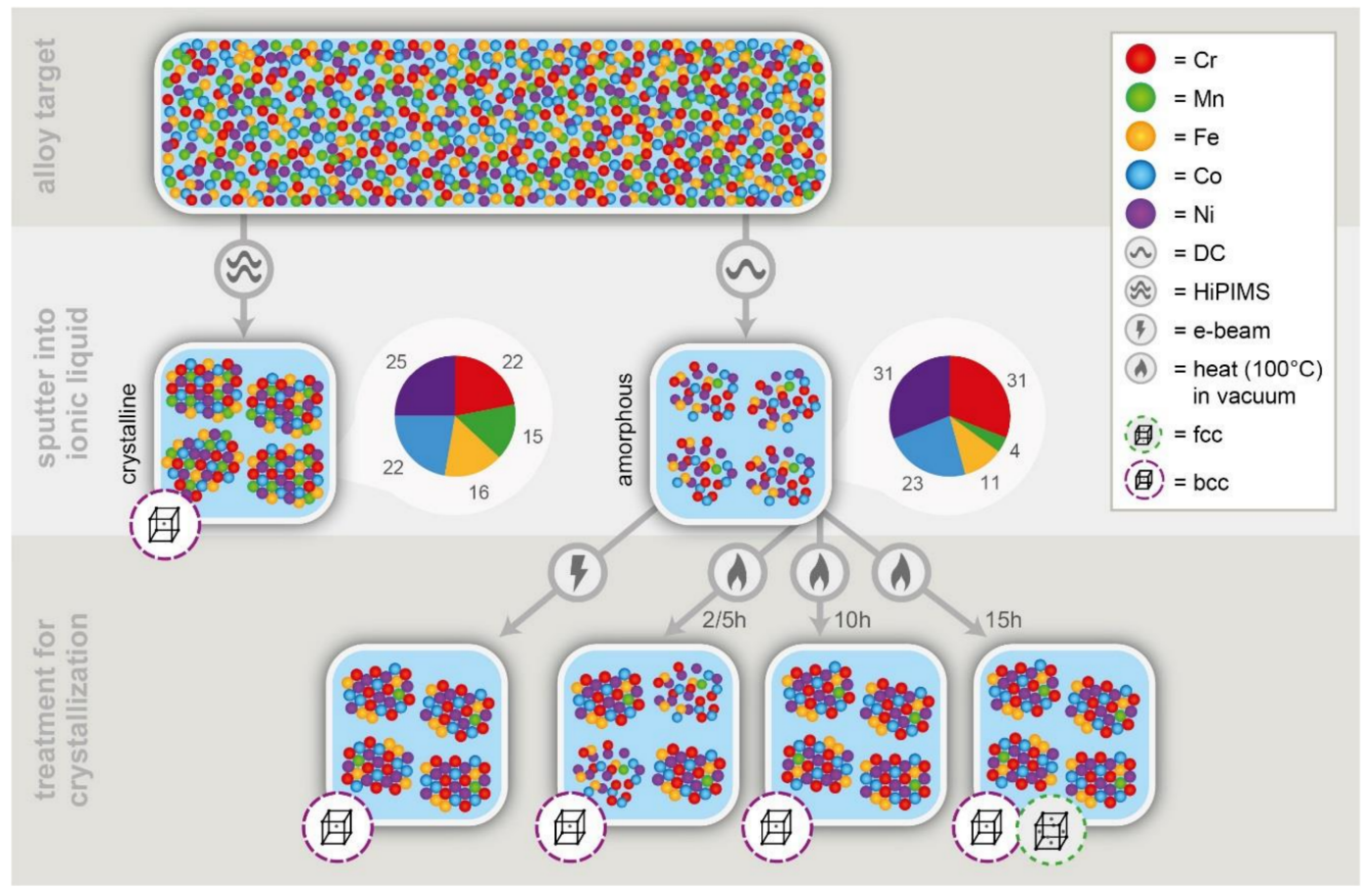

Figure 5. Schematic overview of the different routes used to synthesize amorphous and crystalline multinary NPs. A phase transition from bcc to fcc NPs occurs for ex situ heating at $100{ }^{\circ} \mathrm{C}$ within $15 \mathrm{~h}$.

Crystalline NPs are obtained by the electron beam and annealing treatment or the HiPIMS process. In our study, the HiPIMS process leads directly to crystalline, multinary NPs. The ionized sputtered species are more energetic than sputtered neutrals and exhibit a broad ion energy distribution up to $\sim 100 \mathrm{eV}$. Therefore, the nucleation, morphology, and microstructure can be affected [33] and crystalline phases can be formed at lower temperatures [34-37]. To achieve this, the impinging ion energy and flux have to be tuned by varying the pulse parameters of the HiPIMS discharge.

For HiPIMS and dc sputtering the bcc phase forms, although for the Cantor alloy (equiatomic composition) the fcc crystal structure is expected [38]. STEM-EDS analyses of the dc-as-deposited NPs reveal a low amount of $\mathrm{Mn}$ from 1 at $\%$ to 3 at $\%$, within the NPs. This might lead to a different crystal structure. The low Mn content is related to the utilized sputter technique. With HiPIMS, the sputtered species have a higher energy and thus, might overcome the surface barrier of the IL easier compared to dc sputtering. Therefore, the composition of the NPs is closer to equiatomic; see Table 1. In the case of ex situ heating at $100{ }^{\circ} \mathrm{C}$ for $10 \mathrm{~h}$, the crystallization process is completed and NPs of around 2 to $4 \mathrm{~nm}$ in size are produced in accordance to the previously described processes. EDS yields a low Mn concentration of $1-3$ at $\%$ up to $15 \mathrm{~h}$ annealing. After $15 \mathrm{~h}$ at $100{ }^{\circ} \mathrm{C}$, the system leads to a phase transition from bcc to fcc, which might be correlated to the incorporation of Mn (up to 8 at \%) or temperature. We assume that the changes in composition result from dissolved elements in the solution of the IL after the sputtering process, which are still surrounding the NP and can be incorporated in the crystal lattice during the annealing treatment.

Although the literature reports the fcc structure for the equiatomic Cr-Mn-Fe-Co-Ni system, we find both bcc and fcc structures for the NPs [39] This might be related to the fact that the crystalline structure can differ for NPs and bulk. Furthermore, the compositional deviation from equiatomic composition (see Table 1) might cause differences in crystal structure.

\section{Conclusions}

Different pathways for the controlled synthesis of amorphous or crystalline multinary NPs were developed for the Cr-Mn-Fe-Co-Ni system, utilizing combinatorial co sputtering into the ionic liquid [Bmim][(Tf)2N]. Evidently, applying energy to the amorphous NPs leads to crystallization and a 
larger diameter of the NPs. After in situ electron beam treatment, ex situ annealing up to $15 \mathrm{~h}$ or performing the HiPIMS process, a bcc structure was identified. Moreover, after $15 \mathrm{~h}$ ex situ annealing, a phase transition from bcc to fcc was observed, which can be related to the change in the elemental composition and to the annealing time, which seems to alter the Mn content of the NPs. We also showed the advantages of HiPIMS sputtering technique compared to dc sputtering which provides crystalline multinary NPs in the as-deposited state and a better control of the chemical composition of the sputtered species in the IL, which makes it a promising synthesis method for multinary NPs in general. The results aim to provide a more in-depth understanding of NPs synthesis methods and how the variation of processing parameters allows for tuning of crystallinity, aspired phase formation and particle size which has important implications for the design and controlled synthesis of target-specific NP.

Supplementary Materials: The following data are available online at http:/ / www.mdpi.com/2079-4991/8/11/ 903/s1, Figure S1: Histograms of size distribution of the in-situ TEM crystallization experiment for multinary NPs transferred on a carbon gold grid from the IL (a) shows the amorphous state of the NPs in the initial state $(1.7 \pm 0.2 \mathrm{~nm})(\mathrm{b})$ after 40 min electron beam irradiation $(2.6 \pm 0.3 \mathrm{~nm})$. Figure S2: Histograms of the size distribution of the ex-situ crystallization experiment for multinary NPs at $100{ }^{\circ} \mathrm{C}$ under vacuum (30 Pa) transferred on a carbon coated gold grid from the ionic liquid $2 \mathrm{~h}(1.9 \pm 0.2 \mathrm{~nm}), 10 \mathrm{~h}(2.4 \pm 0.4 \mathrm{~nm})$ and $15 \mathrm{~h}(4.8 \pm 0.8 \mathrm{~nm})$. Figure S3: Histogram of the size distribution of HiPIMS sputtered multinary NPs $(3.2 \pm 0.5 \mathrm{~nm})$ transferred on a carbon grid from the ionic liquid.

Author Contributions: A.G.-M. designed and carried out the TEM, STEM and EDS measurements, in-situ crystallization experiments and phase transition studies. H.M. synthesized multinary nanoparticles, pretested and prepared (S)TEM samples and designed the ex-situ crystallization process. D.G. synthesized multinary nanoparticles especially for the HIPIMs samples. T.L. carried out the ICP-MS measurements. W.S., A.L., and C.S. provided scientific discussion and contribution in writing the manuscript. All co-authors contributed with data interpretation.

Acknowledgments: This work was funded by the German Science Foundation (DFG) via the project SCHE 634/21 1 and LU1175/23-1 as well as the BMBF project NEMEZU (03SF0497B). The HiPIMS power supply was funded by SFB TR 87 and the alloy sputter target Cr-Mn-Fe-Co-Ni was kindly received from Guillaume Laplanche (RUB). We thank Martin Trautmann for his contribution to the ICP-MS measurements and Creative Studio Lena Heel for the graphical abstract and Figure 5.

Conflicts of Interest: The authors declare no conflict of interest.

\section{References}

1. Stepanov, A.L. Nonlinear optical properties of metal nanoparticles in silicate glass. Glas Nanocomposites 2016, 165-179.

2. Li, M.; Cushing, S.K.; Wu, N. Plasmon-enhanced optical sensors: A review. Analyst 2014, 140, $386-406$. [CrossRef] [PubMed]

3. Wu, W. Inorganic nanomaterials for printed electronics: A review. Nanoscale 2017, 9, 7342-7372. [CrossRef] [PubMed]

4. Scholten, J.D.; Leal, C.; Dupont, J. Transition metal nanoparticle catalysis in ionic liquids. ACS Catal. 2012, 2, 184-200. [CrossRef]

5. Stark, W.J.; Stoessel, P.R.; Wohlleben, W.; Hafner, A. Industrial applications of nanoparticles. Chem. Soc. Rev. 2015, 44, 5793-5805. [CrossRef] [PubMed]

6. Ye, Y.F.; Wang, Q.; Lu, J.; Liu, C.T.; Yang, Y. High-entropy alloy: challenges and prospects. Mater. Today 2016, 19, 349-362. [CrossRef]

7. Tsai, M.H.; Yeh, J.W. High-entropy alloys: A critical review. Mater. Res. Lett. 2014, 2, 107-123. [CrossRef]

8. Guo, S.; Liu, C.T. Phase stability in high entropy alloys: Formation of solid-solution phase or amorphous phase. Prog. Nat. Sci. Mater. Int. 2011, 21, 433-446. [CrossRef]

9. Goesmann, H.; Feldmann, C. Nanoparticulate functional materials. Angewandte 2010, 49, $1362-1395$. [CrossRef] [PubMed]

10. Lazarus, L.L.; Riche, C.T.; Malmstadt, N.; Brutchey, R.L. Effect of ionic liquid impurities on the synthesis of silver nanoparticles. Langmuir 2012, 28, 15987-15993. [CrossRef] [PubMed]

11. Chen, C.; Liu, X.; Hedrick, J.L.; Xie, Z.; Wang, S.; Lin, Q.-Y.; Hersam, M.C.; Dravid, V.P.; Mirkin, C.A. Polyelemental nanoparticle libraries. Science 2016, 352, 1565-1569. [CrossRef] [PubMed] 
12. Torimoto, T.; Kameyama, T.; Kuwabata, S. Top-down synthesis methods for nanoscale catalysts. Nanocatalysis Ion. Liq. 2016, 171-205.

13. He, Z.; Alexandridis, P. Nanoparticles in ionic liquids: Interactions and organization. Phys. Chem. Chem. Phys. 2015, 17, 18238-18261. [CrossRef] [PubMed]

14. Wender, H.; Migowski, P.; Feil, A.F.; Teixeira, S.R.; Dupont, J. Sputtering deposition of nanoparticles onto liquid substrates: Recent advances and future trends. Coord. Chem. Rev. 2013, 257, 2468-2483. [CrossRef]

15. Torimoto, T.; Okazaki, K.; Kiyama, T.; Hirahara, K. Sputter deposition onto ionic liquids: Simple and clean synthesis of highly dispersed ultrafine metal nanoparticles. Appl. Phys. Lett. 2006, 84, 243117. [CrossRef]

16. Meyer, H.; Meischein, M.; Ludwig, A. Rapid assessment of sputtered nanoparticle ionic liquid combinations. ACS Comb. Sci. 2018, 20, 243-250. [CrossRef] [PubMed]

17. Vanecht, E.; Binnemans, K.; Seo, J.W.; Stappers, L.; Fransaer, J. Growth of sputter-deposited gold nanoparticles in ionic liquids. Phys. Chem. Chem. Phys. 2011, 13, 13565-13571. [CrossRef] [PubMed]

18. Suzuki, T.; Okazaki, K.; Kiyama, T.; Kuwabata, S.; Torimoto, T. A facile synthesis of AuAg alloy nanoparticles using a chemical reaction induced by sputter deposition of metal onto ionic liquids. Electrochemistry 2009, 77, 636-638. [CrossRef]

19. Suzuki, S.; Suzuki, T.; Tomita, Y.; Hirano, M.; Okazaki, K.I.; Kuwabata, S.; Torimoto, T. Compositional control of AuPt nanoparticles synthesized in ionic liquids by the sputter deposition technique. Cryst. Eng. Comm. 2012, 14, 4922-4926. [CrossRef]

20. Khatri, O.P.; Adachi, K.; Murase, K.; Okazaki, K.; Torimoto, T.; Tanaka, N.; Kuwabata, S.; Sugimura, H. Self-assembly of ionic liquid (BMI-PF6)-stabilized gold nanoparticles on a silicon surface: Chemical and structural aspects. Langmuir. 2008, 24, 7785-7792. [CrossRef] [PubMed]

21. Okazaki, K.; Kiyama, T.; Suzuki, T.; Kuwabata, S.; Torimoto, T. Thermally induced self-assembly of gold nanoparticles sputter-deposited in ionic liquids on highly ordered pyrolytic graphite surfaces. Chem. Lett. 2009, 38, 330-331. [CrossRef]

22. Technol, J.E.S.; Cha, I.Y.; Yoo, S.J.; Jang, J.H. Recent progress in nanoparticle synthesis via liquid medium sputtering and its applications. J. Phys. Chem. 2016, 7, 13-26.

23. König, D.; Richter, K.; Siegel, A.; Mudring, A.; Ludwig, A. High-throughput fabrication of Au-Cu nanoparticle libraries by combinatorial sputtering in ionic liquids. Adv. Funct. Mater. 2014, 24, 2049-2056. [CrossRef]

24. Purposes, C. Bimetallic nanoparticles in alternative solvents for catalytic purposes. Catalysts 2017, 7, 207.

25. Wender, H.; De Oliveira, L.F.; Migowski, P.; Feil, A.F.; Lissner, E.; Prechtl, M.H.G.; Teixeira, S.R.; Dupont, J. Ionic liquid surface composition controls the size of gold nanoparticles prepared by sputtering deposition. J. Phys. Chem. 2010, 114, 11764-11768. [CrossRef]

26. Bohlmark, J.; Alami, J.; Christou, C.; Ehiasarian, A.P.; Helmersson, U. Ionization of sputtered metals in high power pulsed magnetron sputtering. J. Vac. Sci. Technol. 2005, 23, 18-22. [CrossRef]

27. Löffler, T.; Meyer, H.; Savan, A.; Wilde, P.; Garzón-Manjón, A.; Chen, Y.T.; Ventosa, E.; Scheu, C.; Ludwig, A.; Schuhmann, W. Discovery of a multinary nobel metal free oxygen reduction catalyst. Advanced Energy Mater. 2018. [CrossRef]

28. Zhang, T.; Song, Z.; Sun, M.; Liu, B.; Feng, S.; Chen, B. Investigation of electron beam induced phase change in $\mathrm{Si}_{2} \mathrm{Sb}_{2} \mathrm{Te}_{5}$ material. Appl. Phys. A. 2008, 90, 451-455. [CrossRef]

29. Williams, D.B.; Carter, C.B. Transmission Electron Microscopy: A Textbook for Materials Science, 2nd Edition; Springer Science+Business Media: New York, NY, USA, 2009.

30. Lv, Z.Y.; Liu, X.J.; Jia, B.; Wang, H.; Wu, Y.; Lu, Z.P. Development of a novel high- entropy alloy with eminent efficiency of degrading azo dye solutions. Sci. Rep. 2016, 6, 34213. [CrossRef] [PubMed]

31. Mandegarzad, S.; Raoof, J.B.; Hosseini, S.R.; Ojani, R. Cu-Pt bimetallic nanoparticles supported metal organic framework-derived nanoporous carbon as a catalyst for hydrogen evolution reaction. Electrochim. Acta 2016, 190, 729-736. [CrossRef]

32. Liu, J.; Cao, J.; Huang, Q.; Li, X.; Zou, Z.; Yang, H. Methanol oxidation on carbon-supported Pt-Ru-Ni ternary nanoparticle electrocatalysts. J. Power Sources 2008, 175, 159-165. [CrossRef]

33. Alami, J.; Eklund, P.; Andersson, J.M.; Lattemann, M.; Wallin, E.; Bohlmark, J.; Persson, P.; Helmersson, U. Phase tailoring of Ta thin films by highly ionized pulsed magnetron sputtering. Thin Solid Films 2007, 515, 3434-3438. [CrossRef] 
34. Wallin, E.; Selinder, T.I.; Elfwing, M.; Helmersson, U. Synthesis of $\alpha-\mathrm{Al}_{2} \mathrm{O}_{3}$ thin films using reactive high-power impulse magnetron sputtering. EPL 2008, 82, 36002 1-5. [CrossRef]

35. Selinder, T.I.; Coronel, E.; Wallin, E.; Helmersson, U. $\alpha$-Alumina coatings on WC/Co substrates by physical vapor deposition. Int. J. Refract. Met. Hard Mater. 2009, 27, 507-512. [CrossRef]

36. Alami, J.; Sarakinos, K.; Uslu, F.; Klever, C.; Dukwen, J.; Wuttig, M. On the phase formation of titanium oxide films grown by reactive high power pulsed magnetron sputtering. J. Phys. D Appl. Phys. 2009, 42, 115204. [CrossRef]

37. Aiempanakit, M.; Helmersson, U.; Aijaz, A.; Larsson, P.; Magnusson, R.; Jensen, J.; Kubart, T. Effect of peak power in reactive high power impulse magnetron sputtering of titanium dioxide. Surf. Coat. Technol. 2011, 205, 4828-4831. [CrossRef]

38. Cantor, B.; Chang, I.T.H.; Knight, P.; Vincent, A.J.B. Microstructural development in equiatomic multicomponent alloys. Mater. Sci. Eng. A 2004, 375, 213-218. [CrossRef]

39. Kauffmann, A.; Stüber, M.; Leiste, H.; Ulrich, S.; Schlabach, S.; Vinga, D.; Seils, S.; Gorr, B.; Chen, H.; Seifert, H.; et al. Combinatorial exploration of the high entropy alloy system Co-Cr-Fe-Mn-Ni. Surf. Coat. Technol. 2017, 325, 174-180. [CrossRef]

(C) 2018 by the authors. Licensee MDPI, Basel, Switzerland. This article is an open access article distributed under the terms and conditions of the Creative Commons Attribution (CC BY) license (http://creativecommons.org/licenses/by/4.0/). 